\title{
Can the BMI-Based Dose Regimen be Used to Reduce Injection Activity and to Obtain a Constant Image Quality in Oncological Patients by 18F-FDG Total-Body PET/CT Imaging?
}

Jie Xiao ( $\square$ xiaojie_fdu@163.com )

Zhongshan Hospital Fudan University

Haojun Yu

Zhongshan Hospital Fudan University

Xiuli Sui

Zhongshan Hospital Fudan University

Yan $\mathrm{Hu}$

Zhongshan Hospital Fudan University

Cao Yanyan

Zhongshan Hospital Fudan University

Guobing Liu

Zhongshan Hospital Fudan University

Yiqiu Zhang

Zhongshan Hospital Fudan University

Pengcheng Hu

Zhongshan Hospital Fudan University Department of Nuclear Medicine

\section{Ying Wang}

Central Research Institute of united Imaging Healthcare

Chenwei Li

Central Research Institute of united Imaging Healthcare

Baixuan Xu

301 Military Hospital: Chinese PLA General Hospital

Hongcheng Shi

Zhongshan Hospital Fudan University

\section{Research Article}

Keywords: Image quality, dose optimization, patient size, total-body PET, FDG, PET/CT imaging

Posted Date: April 14th, 2021 
DOl: https://doi.org/10.21203/rs.3.rs-402099/v1

License: (c) (1) This work is licensed under a Creative Commons Attribution 4.0 International License. Read Full License

Version of Record: A version of this preprint was published at European Journal of Nuclear Medicine and Molecular Imaging on June 29th, 2021. See the published version at https://doi.org/10.1007/s00259021-05462-5. 
Can the BMI-based dose regimen be used to reduce injection activity and to obtain a constant image quality in oncological patients by ${ }^{18}$ F-FDG total-body PET/CT imaging?

Jie Xiao ${ }^{1,2,3}$, Haojun $\mathrm{Yu}^{1,2,3}$, Xiuli Sui ${ }^{1,2,3}$, Yan $\mathrm{Hu}^{1,2,3}$, Yanyan $\mathrm{Cao}^{1,2,3}$, Guobing Liu ${ }^{1,2,3}$, Yiqiu Zhang ${ }^{1,2,3}$, Pengcheng Hu${ }^{1,2,3}$, Ying Wang ${ }^{4}$, Chenwei $\mathrm{Li}^{4}$, Baixuan $\mathrm{Xu}^{5 *}$, Hongcheng Shi ${ }^{1,2,3^{*}}$

${ }^{1}$ Department of Nuclear Medicine, Zhongshan Hospital, Fudan University, 180 Fenglin Road, Shanghai, 200032, China.

${ }^{2}$ Institute of Nuclear Medicine, Fudan University, Shanghai, 200032, China.

${ }^{3}$ Shanghai Institute of Medical Imaging, Shanghai, 200032, China.

${ }^{4}$ Central Research Institute of United Imaging Healthcare, Shanghai, 201807, China.

${ }^{5}$. Department of Nuclear Medicine, Chinese PLA General Hospital, Beijing 100853, China.

* Corresponding to:

Baixuan Xu, E-mail address: xbx301@163.com

Hongcheng Shi, E-mail address: Shi.hongcheng@zs-hospital.sh.cn 


\section{Abstract}

Purpose PET image quality is influenced by the patient size according to the current guideline. The purpose was to propose an optimized dose regimen to yield a constant image quality independent of patient size to meet the clinical needs.

Methods A first patient cohort of 78 consecutives for oncological patients (59.7 \pm 13.7 years) who underwent a total-body PET/CT scan were retrospectively enrolled to develop the regimen. The patients were equally distributed in four BMI groups according to

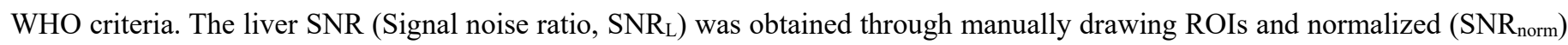
by the injected activity and acquisition time. And fits of $\mathrm{SNR}_{\text {norm }}$ against different patient-dependent parameters were performed to determine the best correlating parameter and fit method. A qualitative assessment on image quality was performed using a 5-point Likert scale to determine the acceptable threshold of $\mathrm{SNR}_{\mathrm{L}}$. And thus, an optimized regimen was proposed and validated by a second patient cohort with prospectively enrolled 38 oncological patients.

Results The linear fit showed $\mathrm{SNR}_{\text {norm }}$ was the strongest correlation $\left(\mathrm{R}^{2}=0.69\right)$ with the BMI than other patient-dependent parameters. The qualitative assessment revealed a $\mathrm{SNR}_{\mathrm{L}}$ of 14.0 as a threshold to achieve a sufficient image quality. The optimized dose regimen was determined as a quadratic relation with BMI: Injected activity $=39.2 \mathrm{MBq} /(-0.03 * \mathrm{BMI}+1.49)^{2}$. In the validation study, the $\mathrm{SNR}_{\mathrm{L}}$ no longer decreased with the increase of BMI. There was no significant difference of the image quality, the $\mathrm{SNR}_{\mathrm{L}}$, between different BMI groups $(p>0.05)$. In addition, the injected activity was reduced by $75.6 \pm 2.9 \%, 72.1 \pm 4.0 \%, 67.1 \pm 4.4 \%$ and $64.8 \pm 3.5 \%$ compared to the first cohort for the four BMI groups, respectively.

Conclusion The study recommended a quadratic relation between the ${ }^{18} \mathrm{~F}-\mathrm{FDG}$ injected activity and the patient's BMI and propose a regimen for total-body PET imaging. In the regimen, the image quality can maintain in a constant level independent of patient size and meet the clinical requirement even with a reduced injected activity.

Keywords Image quality, dose optimization, patient size, total-body PET, FDG, PET/CT imaging, 
F-18-fluorodeoxyglucose Positron emission tomography/Computed Tomography $\left({ }^{18} \mathrm{~F}\right.$-FDG PET/CT) is being widely used in tumor diagnosis, staging, restaging, and response evaluation. Meanwhile, this hybrid imaging modality has shown the potential value in prognosis prediction, image-guided biopsy, providing both anatomic and functional information for clinical management [1-5]. The total-body PET/CT, with an increased geometric overage to encompass the entire body, can dramatically improve the PET sensitivity by a factor of about 40 over existing PET scanners for imaging the entire body. This predicted gain in sensitivity has various implications, such as to improve the image quality to reconstruct images at higher resolution and allow detection of smaller or lowercontrast structures. In addition, it can be used to reduce the injected activity, or to reduce PET acquisition duration while maintaining the image quality [6].

Previously, our team has conducted a series of research focusing on image quality. Zhang et. al [7] found that oncological patients with the injected activity of $4.4 \mathrm{MBq} / \mathrm{kg}$ could acquire sufficient and acceptable subjective image quality for an acquisition time of $30 \mathrm{~s}$ and $60 \mathrm{~s}$. To explore whether the low-dose activity could obtain an acceptable image quality, the ${ }^{18} \mathrm{~F}-\mathrm{FDG}$ activity of 0.58 $\mathrm{MBq} / \mathrm{kg}$ was injected into a subject weighing $43.5 \mathrm{~kg}$ (total dose: $25 \mathrm{MBq}, 15.7 \%$ of the conventional dose (161 MBq) $50 \mathrm{~min}$ after injection with an acquisition time of $10 \mathrm{~min}$. This generated a feasible image for diagnosis [8]. Furthermore, our previous results showed that total-body PET/CT with half-dose $(1.85 \mathrm{MBq} / \mathrm{kg}){ }^{18} \mathrm{~F}-\mathrm{FDG}$ of $2 \mathrm{~min}$ acquisition, could achieve an equivalent image quality to that of whole-body PET/CT with full-dose (3.7 MBq/kg) in lung cancer [9]. Recently, Liu et.al reported that dynamic PET imaging of ultra-low-dose $(0.37 \mathrm{MBq} / \mathrm{kg})$ injected activity achieved relevant kinetic metrics of ${ }^{18} \mathrm{~F}-\mathrm{FDG}$ and comparable image contrast with full-dose imaging [10].

However, these studies have all adopted a weight-based injected activity as recommended in the EANM Guideline [11]. It is wellknown that a weight-based dose regimen had several deficiencies for a stable and reliable PET image, especially in obese patients with a significantly high amount of body fat resulting in low FDG accumulation [12]. Based on the above-mentioned studies, the purpose of this study was to further investigate the influence of patient-dependent parameters on image quality and propose a personalized and a more universal dose regimen to yield a more constant image quality.

\section{Materials and Methods}

\section{Patients}

The study included two cohorts who underwent a total-body PET/CT examination in our department. The first patient cohort, consisting of 78 consecutive patients who underwent a total-body PET/CT examination referred for oncological diagnosis and/or staging from September 2019 to July 2020, was studied to explore a new dose regimen based on different patient-dependent parameters. They were randomly selected from the database in our department. Patients with severe fatty liver, cirrhosis and multiple metastasis were excluded. The included 78 patients were equally distributed in each body mass index (BMI) group according to the criteria of the World Health Organization [13], with 20 patients in the underweight group, 19 patients in the normal-weight group, 19 patients in the overweight group and 20 patients in the obese group. Subsequently, the second patient cohort consisting of 38 patients with known or suspected malignancy were prospectively enrolled to validate the proposed dose regimen, including 7 patients, 10 patients, 11 patients and 10 patients for each BMI group, respectively. Exclusion criteria for the second patient cohort 
are those with diabetes, or younger than 18 years old. In both cohorts, patient demographic characteristics were extracted from the database, including sex, age, weight $(\mathrm{BM})$, and height $(\mathrm{H}) . \mathrm{BMI}\left(\mathrm{kg} / \mathrm{m}^{2}\right)$ was calculated by dividing the body mass $(\mathrm{kg})$ by the square of height (m). Considering human body composition, lean body weight (LBW, kg), fat mass (FM, kg) were also investigated as patient-dependent parameters in the study. LBM for male and female as well as FM was calculated as follows (Eqs. 1-3, respectively) [14].

$$
\begin{array}{ll}
L B W_{\text {male }}=28.27 \times \mathrm{H}+0.359 \times \mathrm{BM}-0.032 \times \text { age }-21.83 & \text { Eq. } 1 \\
L B W_{\text {female }}=26.12 \times \mathrm{H}+0.253 \times \mathrm{BM}-0.022 \times \text { age }-19.58 & \text { Eq. } 2 \\
\mathrm{FM}=\mathrm{BM}-\mathrm{LBW} & \text { Eq. } 3
\end{array}
$$

In Eq. 1 and 2, LBW is the lean body mass in kilograms, $\mathrm{H}$ is the height in meters, BM is the body weight in kilograms, and age is in years. In Eq. 3, FM is the fat mass in kilograms, BM is the body weight in kilograms, and LBW is the lean body mass in kilograms. The use of patients' information included in this study according to the standard procedures were in accordance with the institutional ethical standards. Informed consent was waived to the patients in the first cohort waived due to the retrospective nature and all patients in the second cohort signed an informed consent prior to the PET/CT scan.

\section{PET/CT examination}

All patients were instructed to fast and avoid strenuous exercise at least $6 \mathrm{~h}$ prior to the ${ }^{18} \mathrm{~F}-\mathrm{FDG}$ injection, and blood glucose level was measured and recorded. In the study on the first patient cohort, a bolus injection of ${ }^{18} \mathrm{~F}-\mathrm{FDG}(3.7 \mathrm{MBq} / \mathrm{kg}) \mathrm{was}$ intravenously administered. And in the subsequent study of the second patient cohort, the injection was strictly following the proposed dose regimen. All mages were acquired on a total-body PET/CT scanner (uEXPLORER, United Imaging Healthcare, Shanghai, China). CT was performed before PET for attenuation correction and anatomical localization with a dose modulation technique. Subsequently, a total-body PET imaging was performed using a list-mode PET acquisition with a 5 min duration with arms down positioning.

The acquisition time was segmented into 30s, 45s, 60s, and 120s from the 300s list-mode raw data, referred as G30, G45, G60, G120, and G300. All the PET images were reconstructed using a 3D ordered-subset expectation maximization algorithm (3D-OSEM) with the following parameters: 3 iterations, 20 subsets, a matrix of $192 \times 192$, slice thickness of $1.443 \mathrm{~mm}$, time of flight (TOF) and point spread function modeling (PSF). A Gaussian filter with a Full Width Half Maximum of 3 mm was applied to the reconstructed images.

\section{Image analysis}

In PET studies, the signal-to-noise ratio $(\mathrm{SNR})$ in the liver $\left(\mathrm{SNR}_{\mathrm{L}}\right)$ was used as a measure of image quality as it is the organ with a relatively homogeneous uptake of FDG in the human body. It is well-known that various factors, such as the patient weight, injected activity and acquisition time, can impact the $\mathrm{SNR}_{\mathrm{L}}$.

For a given situation on a PET scanner, SNR in PET images is dominated by the Poisson statistics inherent in radionuclide decay detection and is proportional to the square root of the detected events. In the first-order approximation we expect that [7]. 
Where $\mathrm{k}$ is a constant, $\mathrm{S}$ is the effective sensitivity of the scanner, $\mathrm{A}$ is the injected activity $(\mathrm{MBq})$ and $\mathrm{t}$ is the acquisition time per bed position ( $\mathrm{min})$. Here, the dose-time product (DTP $(\mathrm{MBq} \cdot \mathrm{min}))$ is the product of the injected activity (MBq) and the acquisition time per bed position (min). If the SNR in the liver is normalized by the square root of the DTP, it can be assumed to be independent of the injected activity and the acquisition time (Eq. 5) [16]. Therefore, $\mathrm{SNR}_{\text {norm }}$ can be regarded as a function of various patientdependent parameters and investigated in the study.

$$
S N R_{n o r m}=\frac{S N R_{L}}{\sqrt{D T P}}
$$

In the first part of the study, the slice with the largest cross section in the liver on the CT transverse slice was determined. In the corresponding PET slice and two adjacent slices, a circular region of interest (ROI) with a diameter of $2.0 \pm 0.1 \mathrm{~cm}$ was manually drawn in a lesion-free and homogenous region of the right liver lobe with care to avoid large blood vessels and partial volume effects (supplementary Figure 1). The ROIs were identical in all three slices. Liver $\mathrm{SUV}_{\text {mean }}$ and its standard deviation (SD) were measured and recorded, and determined as the average values of three ROIs. The $\mathrm{SNR}_{\mathrm{L}}$ was obtained by dividing the $\mathrm{SUV}$ mean by the SD (Eq. 6) $[15]$.

$$
\mathrm{SNR}_{L}=\frac{S U V_{\text {mean }}}{S D}
$$

Both linear and exponential fits were performed with the $\mathrm{SNR}_{\text {norm }}$ and patient-dependent parameters, and the highest coefficient of determination $\left(\mathrm{R}^{2}\right)$ was used to determine the best-correlated parameter. In addition, the relation between the $\mathrm{SNR}_{\text {norm }}$ and the patient-dependent parameters, referred as $\mathrm{SNR}_{\text {fit }}$ were obtained from the fit function (Eq. 7).

$$
S N R_{\text {norm }} \approx S N R_{f i t}=a(p+b)^{c}
$$

Where $\mathrm{p}$ indicated the best-correlated parameter, $\mathrm{a}, \mathrm{b}$ and $\mathrm{c}$ were constants derived from the fit function.

In order to determine the acceptable $\mathrm{SNR}_{\mathrm{L}}$ threshold $\left(\mathrm{SNR}_{\mathrm{acc}}\right)$, a qualitative analysis on image quality was performed. The image quality was qualitatively evaluated by two experienced nuclear medicine physicians on a dedicated workstation (uWI, United Imaging Healthcare, Shanghai, China). Both readers were blinded to the patient's history, injected activity and the acquisition time. Image was scored based on a 5-points Likert scale to assess the overall image quality (1=non-diagnostic image quality; $2=$ poor image quality; $3=$ moderate image quality; 4=good image quality; $5=$ excellent image quality), as shown in Fig. 3 . The score of 3 was equivalent to the image quality to meet the clinical need in our department and served as the reference to determine the acceptable $\mathrm{SNR}_{\mathrm{L}}$ threshold $\left(\mathrm{SNR}_{\mathrm{acc}}\right)$. The $\mathrm{SNR}_{\mathrm{acc}}$ was obtained by calculating the mean of $\mathrm{SNR}_{\mathrm{L}}$ from all the images scored with 3 points. Finally, the dose regimen was determined as follows (Eq. 8).

$$
\text { Injected activity }=\frac{\left({ }^{S N R_{a c c}} / S N R_{f i t}\right)^{2}}{t}
$$

Where $t$ indicated the acquisition duration, and $\mathrm{SNR}_{\mathrm{fit}}$ was the function obtained from the fit function.

Subsequently, the proposed dose regimen for was validated with a newly enrolled patient cohort. In the validation, the patient was injected with the ${ }^{18}$ F-FDG strictly following the proposed regimen. And image quality was assessed qualitatively using the same criteria by the same nuclear medicine physicians. A subsequent quantitative analysis was performed to compare the liver $\mathrm{SNR}_{\mathrm{L}}$, $\mathrm{SUV}_{\text {mean }}$ and SD between the two cohorts. In order to assess the lesion contrast, a lesion with the maximum uptake was selected to 
calculate the target-to-background ratio (TBR). A volume of interest (VOI) was manually drawn on each selected lesion and SUV $\mathrm{V}_{\text {max }}$ was obtained. The lesion TBR can be calculated by dividing its $\mathrm{SUV}_{\max }$ by the $\mathrm{SUV}_{\mathrm{L}}$.

$$
\mathrm{TBR}=\frac{\text { lesionsUV } \max }{S U V_{L}}
$$

\section{Statistical analysis}

All statistical analysis was performed using IBM SPSS Statistics Version 26 (IBM Inc., Chicago, IL, USA) and Prism 8 (GraphPad Software Inc., San Diego, California, USA). Data were described as mean \pm SD. Differences in quantitative variables were assessed by analysis of variance (ANOVA) with post hoc Bonferroni adjustment for pairwise comparison. Independent $t$ test was performed to compare the quantitative variables from the two cohorts. Categorical variables were compared using the Chi-square test. Cohen's kappa analysis was performed to evaluate the inter-reader agreement. Results were considered statistically different if $\mathrm{p}$ is less than 0.05 .

\section{Results}

\section{Patient characteristics}

The demographic characteristics of the two patient cohorts were listed in Table 1. In the first patient cohort to develop the dose regimen, a total number of 78 oncological patients (Male: 51/Female: 27 ) with an average age of 59.7 \pm 13.7 years (range: 19-81 years), were retrospectively studied. The location of the primary tumor of the enrolled patients included 4 patients in the head and neck, 19 in the chest, 54 in the abdomen and pelvic cavity, and 1 in the retroperitoneum. In the second patient cohort to validate the proposed dose regimen, 38 patients (Male: 29/Female: 9) with an average age of $61.3 \pm 14.1$ years were enrolled, including 1 patient with primary tumor located in the head and neck, 12 in the chest, and 25 in the abdomen and pelvic cavity. The blood glucose level for all the enrolled patients was under $6.1 \mathrm{mmol} / \mathrm{L}$ with an uptake time of $74.9 \pm 21.0 \mathrm{~min}$ (range: $45.0-121.0 \mathrm{~min}$ ) and $77.3 \pm 16.1$ min (range: 46.0-120.0 $\mathrm{min}$ ) for the two cohorts, respectively. And there were no significant differences in gender, age, BM, weight, BMI, LBM, FM, BSA, blood glucose level and uptake time between the two patient cohorts (all $p>0.05$ ).

\section{The development of the dose regimen}

The $\mathrm{SNR}_{\mathrm{L}}$ increased along with the increase of acquisition duration with a significant difference to that in G300 (as shown Fig. 1). Moreover, $\mathrm{SNR}_{\mathrm{L}}$ differed between BMI groups, as illustrated in Table 2. The $\mathrm{SNR}_{\mathrm{L}}$ decreased along with the increase of the BMI groups for all the acquisition, as observed in the clinical practice. As expected, the $\mathrm{SNR}_{\mathrm{L}}$ of the obese group was significantly lower than any other groups (all $p<0.05$ ). The $\mathrm{SNR}_{\mathrm{L}}$ in the obese group with a 5-min acquisition was comparable to that in the normal weight group with a 2 -min acquisition $(18.2 \pm 3.4$ vs. $18.1 \pm 4.2, p=0.37)$.

The $\mathrm{SNR}_{\text {norm, }}$ the normalized SNR $\mathrm{L}$, was fitted with the different patient-dependent parameters using a linear and non-linear method, as illustrated in Fig. 2. It was found that the $\mathrm{SNR}_{\text {norm }}$ best fitted with BMI with a linear fit function, with the highest coefficient $\left(\mathrm{R}^{2}\right.$ $=0.69)$ and slightly lower coefficient in a non-linear fit with BMI $\left(\mathrm{R}^{2}=0.68\right)$. Therefore, BMI was determined as the best-correlated parameter, with a linear fit function (Eq. 10). 


$$
S N R_{\text {norm }}=-0.03 * B M I+1.49
$$

The overall image quality of G30, G45, G60 and G120 were scored with $2.09 \pm 0.29,2.47 \pm 0.50,2.97 \pm 0.48$ and $3.82 \pm 0.38$, respectively, as presented in Table 3. The inter-reader agreement of overall image quality showed a substantial result with a kappa value of $0.93,0.99,0.95$ and 0.94 for each acquisition duration, respectively. A SNR acc value of 14.0 was obtained by calculating the average of the SNR $\mathrm{L}_{\mathrm{L}}$ of all the images with a score of 3 points. Therefore, for a given acquisition duration of 5 minutes, the new dose regimen can be determined as (Eq. 11):

$$
\text { Injected Activity }(\mathrm{MBq})=\frac{39.2}{(-0.03 * B M I+1.49)^{2}}
$$

\section{Validation of the proposed regimen}

The new dose regimen was validated with the second patient cohort consisted of 38 oncological patients both qualitatively and quantitatively. In the qualitative analysis, there was no significant difference between BMI groups (as shown in Fig. 4), indicating a constant visual image quality. Comparing with that in the first patient cohort, the image quality of the patients with a BMI no less than 25 was improved, as illustrated in Figure 5 showing a scan of patients with a BMI of 25 and 30, respectively.

In the quantitative analysis, there were no significant differences in liver $\mathrm{SUV}_{\text {mean }}$ between the patient cohorts $(\mathrm{p}>0.05$, as shown in Table 4), indicating a consistent quantification. The $\mathrm{SNR}_{\mathrm{L}}$ of the all the enrolled patients was plotted as the BMI, as shown in Fig. 6a. The $\mathrm{SNR}_{\mathrm{L}}$ in the validation cohort showed no tendency of decrease with BMI, indicating a stable image quality independent of patient size. In addition, there was no significant difference of the $\mathrm{SNR}_{\mathrm{L}}$ between different BMI groups, as shown in Fig. $6 \mathrm{~b}$. With regards to the selected lesions, the lesion $\mathrm{SUV}_{\max }$ and $\mathrm{TBR}$ showed no significant difference between the patient cohorts (both $\mathrm{p}>$ 0.05 , as shown in Table 4).

In addition, the injected activity of the two patient cohorts was investigated, as illustrated in Fig. 7. The injection activity was 236.8 5 59.2 MBq (range, 136.9-403.3 MBq), and 79.0 23.1 MBq (range, 40.6-123.0 MBq) for the two cohorts with significant difference $(p<0.0001)$. The average injected activity in the first patient cohort were 177.0 $\pm 15.7 \mathrm{MBq}, 204.8 \pm 21.9 \mathrm{MBq}, 255.7 \pm 26.6$ MBq and 315.3 $\pm 44.3 \mathrm{MBq}$ for the four BMI groups, respectively. However, the average injected activity of the validation cohort was reduced to $43.2 \pm 2.5 \mathrm{MBq}, 68.0 \pm 8.3 \mathrm{MBq}, 84.0 \pm 7.2 \mathrm{MBq}$ and 107.2 $\pm 8.3 \mathrm{MBq}$ for four BMI groups, respectively. Therefore, the overall average activity was reduced by $69.2 \pm 5.4 \%$. In addition, the injected activity was reduced by $75.6 \pm 2.9 \%, 72.1 \pm 4.0 \%$, $67.1 \pm 4.4 \%$ and $64.8 \pm 3.5 \%$ of developing datasets for the four BMI groups with a slight difference.

\section{Discussion}

The current European Association of Nuclear Medicine (EANM) and the Society of Nuclear Medicine and Molecular Imaging (SNMMI) guidelines recommended a linear weight-based regimen for ${ }^{18} \mathrm{~F}$-FDG PET examinations [11, 17]. A quadric relationship between the ${ }^{18} \mathrm{~F}-\mathrm{FDG}$ administered activity, PET acquisition time and patient weight was also described in the guideline [11]. However, when a weight-based regimen is applied, the $\mathrm{SNR}_{\mathrm{L}}$ for the patient weight over $75 \mathrm{~kg}$ will be decreased, indicating a degraded image quality due to excessive attenuation and scatter. And thus, an experienced technician is required to modify the acquisition scheme in the clinical scenario for specific situations, which inevitably complicates the operations. Previous studies 
utilized a higher ${ }^{18}$ F-FDG activity per kilogram for patients with a body mass over $90 \mathrm{~kg}$ to compensate for attenuation, while $\mathrm{SNR}_{\mathrm{L}}$ still decreased with body mass in the overweight group [15]. In previous studies, $\mathrm{SNR}_{\mathrm{L}}$ of 9.6 and 10.0 could yield a good image quality $[15,18]$. Tan et al. reported that the $\mathrm{SNR}_{\mathrm{L}}$ of 11.7 in the half-dose total-body group with a two-min duration was higher than the $\mathrm{SNR}_{\mathrm{L}}$ of 8.3 in full-dose whole-body group. However, in this study, the subjective assessment of image quality found that a $\mathrm{SNR}_{\mathrm{L}}$ of 14.0 could obtain a sufficient image quality. A reason for the higher $\mathrm{SNR}_{\mathrm{L}}$ in this study may be bias from different raters. It has been known that the patient-dependent parameters can influence the PET image quality, which is the initial motivation of this study. The fits of $\mathrm{SNR}_{\text {norm }}$ with different parameters showed that both the quadric and linear fitting with BMI had the highest $\mathrm{R}^{2}$ ( 0.68 and 0.69 , respectively). Other parameters, such as the body mass, height, body mass per height, lean body mass, fat mass and body surface area showed a lower value of $\mathrm{R}^{2}$ than the parameters discussed in previous studies $[15,16]$. Based on the results, a linear fit with BMI was selected. The findings were inconsistent with previous studies which suggested a quadratic dose regimen of body mass. This may be due to the difference of the subjects selected in the study. The body mass of the first patient cohorts ranged from 34 to $110 \mathrm{~kg}$ and the number of enrolled patients for each BMI group was larger than the previous study. Moreover, the population body shape varies with races, which might also induce a bias to the results.

A quadratic relation between the BMI and injected ${ }^{18} \mathrm{~F}-\mathrm{FDG}$ activities was determined in this study, which contributes to more constant image quality not affected by BMI. Although this regimen is less convenient than a linear relation in clinical practice, it can be easily overcome by an automatic calculator or a look-up table. Obviously, the benefit of the new regimen exceeds the difficulties introduced. In the proposed regimen, the injected activity was with a $69.2 \pm 5.4 \%$ reduction compared with that in the weight-based regimen (3.7 MBq/kg). In addition, the injected activity was reduced by $75.6 \pm 2.9 \%, 72.1 \pm 4.0 \%, 67.1 \pm 4.4 \%$ and $64.8 \pm 3.5 \%$ for the underweight, normal weight, overweight and obese patient group, respectively. Additionally, the injected activity of normal weight group $(68.0 \pm 8.3 \mathrm{MBq})$ according to the proposed regimen in our study was lower than the half-dose (119.5 \pm 18.8 MBq) study for lung cancer [9]. It is due to the high sensitivity of the total-body PET scanner which is about 40-fold of that for a conventional PET scanner [11]. This helps to provide the regimen with a reduced injected activity while maintaining the image quality feasible for clinical practice. Obviously, the proposed regimen had limited application in patients due to the methodology. The patients with a BMI $\geq 35$ were not enrolled in the study, even the patients referred a PET/CT scan in our site, were scarce. And thus, extrapolation was simply used to develop the regimen. Due to the mathematical nature of the quadric expression, the injected activity was dramatically increased for patients with. And thus, an upper limit should be determined. Here, we simply investigated the $\mathrm{SNR}_{\mathrm{L}}$ against $\mathrm{BMI}$ in a linear relationship: $\mathrm{SNR}_{\mathrm{L}}=-0.5 * \mathrm{BMI}+33.1$. Since the acceptable of the $\mathrm{SNR}_{\mathrm{L}}$ should be more than 14.0 to meet the need of image quality, the upper limit of BMI was determined as $38.2 \mathrm{~kg} / \mathrm{m}^{2}$. And thus, the proposed regimen may be not feasible for the patient with a BMI larger than the upper limit.

In this study, SNR measured in the liver was selected as a measure to assess the image quality since the liver has a relatively homogeneous uptake of ${ }^{18}$ F-FDG. However, SNR could be influenced by several physiological factors, such as blood glucose levels, uptake time, plasma clearance, drinking water status, etc. Blood glucose levels affects liver uptake of the activity as reported in other studies [19]. To avoid interference, the glucose level was controlled within a normal range (3.9-6.1 mmol/L) for both cohorts. 
Further study should be performed with a consideration on the glucose level in a wider range. Additionally, plasma clearance can be influenced by water consumption before the scan and the distribution of FDG may be influenced. In our clinical practice, although the patients were recommended to drink $0.5-1 \mathrm{~L}$ of water after the injection of FDG, not all the enrolled patients strictly followed this instruction. The uptake time of FDG also influenced the level of plasma clearance and the liver SNR. A previous study reported that liver SUV remains constant if the uptake time is in the range of 50-110 $\mathrm{min}$ [20]. The uptake time varied from 45 to $121 \mathrm{~min}$ in the study, and the results may be biased.

The study has several limitations. Firstly, both the training and the validation cohorts do not include subjects with a BMI larger than 35. Secondly, it is a single-center preliminarily study, and the BMI-based regimen needs to be further validated in a future multicenter study. Thirdly, this study just simplified the regimen as a function of the injected activity. In future study, the combination of personalized acquisition time and injected activity should be further explored.

\section{Conclusion}

The study recommended a quadratic relation between the ${ }^{18} \mathrm{~F}-\mathrm{FDG}$ injected activity and the patient's BMI and propose a regimen for total-body PET imaging. In the regimen, the image quality can maintain in a constant level independent of patient size and meet the clinical requirement even with a reduced injected activity.

\section{Funding}

This study is supported by the Three-year Action Plan of Clinical Skills and Innovation of Shanghai Hospital Development Center (grant number: SHDC2020CR3079B), and Science and Technology Committee of Shanghai Municipality (20DZ2201800), Special Fund for Clinical Research, Young Program of Zhongshan Hospital of Fudan University (2018ZSQN38, 2019ZSYQ28, 2020ZSLC63) young program of Zhongshan Hospital and Shanghai "Rising Stars of Medical Talent"-Youth Development Program (HWJRS2019-720), the Shanghai Municipal Key Clinical Specialty Project (Grant number: SHSLCZDZK03401).

\section{Ethical approval}

All procedures performed in studies involving human participants were in accordance with the ethical standards of the institutional and/or national research committee and with the 1964 Helsinki declaration and its later amendments or comparable ethical standards.

\section{Authors' Contributions}

Jie Xiao was involved in the statistical analysis and manuscript writing. Haojun Yu contributed to data acquisition and reconstructions. Xiuli Sui and Yan Hu contributed to data analyses and image interpretation. Guobing Liu and Yanyan Cao helped with data processing. Yiqiu Zhang and Pengcheng Hu supervised the study. Ying Wang and Chenwei Li were with editing English grammar. Hongcheng Shi and Baixuan Xu designed the study and contributed to editing and reviewing the manuscript. All authors read and approved the final manuscript. 


\section{Conflicts of interest}

Ying Wang and Chenwei Li are employees of the Central Research Institute of United Imaging Healthcare. The other authors who are working in Zhongshan Hospital have full control of the data and declare that they have no conflicts of interest. 


\section{References}

1. Bosch KD, Chicklore S, Cook GJ, Davies AR, Kelly M, Gossage JA, et al. Staging FDG PET-CT changes management in patients with gastric adenocarcinoma who are eligible for radical treatment. Eur J Nucl Med Mol Imaging. 2020;47(4):759767. 10.1007/s00259-019-04429-x.

2. Wang H, Zhao S, Li L, Tian R. Development and validation of an (18)F-FDG PET radiomic model for prognosis prediction in patients with nasal-type extranodal natural killer/T cell lymphoma. Eur Radiol. 2020;30(10):5578-5587. 10.1007/s00330020-06943-1.

3. Broccoli A, Nanni C, Cappelli A, Bacci F, Gasbarrini A, Tabacchi E, et al. Diagnostic accuracy of positron emission tomography/computed tomography-driven biopsy for the diagnosis of lymphoma. Eur J Nucl Med Mol Imaging. 2020;47(13):3058-3065. 10.1007/s00259-020-04913-9.

4. Kang F, Mu W, Gong J, Wang S, Li G, Li G, et al. Integrating manual diagnosis into radiomics for reducing the false positive rate of (18)F-FDG PET/CT diagnosis in patients with suspected lung cancer. Eur J Nucl Med Mol Imaging. 2019;46(13):27702779. 10.1007/s00259-019-04418-0.

5. Lopci E, Mascarin M, Piccardo A, Castello A, Elia C, Guerra L, et al. FDG PET in response evaluation of bulky masses in paediatric Hodgkin's lymphoma (HL) patients enrolled in the Italian AIEOP-LH2004 trial. Eur J Nucl Med Mol Imaging. 2019;46(1):97-106. 10.1007/s00259-018-4155-4.

6. Cherry SR, Jones T, Karp JS, Qi J, Moses WW, Badawi RD. Total-Body PET: Maximizing Sensitivity to Create New Opportunities for Clinical Research and Patient Care. J Nucl Med. 2018;59(1):3-12. doi: 10.2967/jnumed.116.184028.

7. Zhang YQ, Hu PC, Wu RZ, Gu YS, Chen SG, Yu HJ, et al. The image quality, lesion detectability, and acquisition time of 18F-FDG total-body PET/CT in oncological patients. Eur J Nucl Med Mol Imaging. 2020 Oct;47(11):2507-2515. doi: 10.1007/s00259-020-04823-w.

8. Badawi RD, Shi H, Hu P, Chen S, Xu T, Price PM, et al. First Human Imaging Studies with the EXPLORER Total-Body PET Scanner. J Nucl Med. 2019;60(3):299-303. 10.2967/jnumed.119.226498.

9. Tan H, Sui X, Yin H, Yu H, Gu Y, Chen S, et al. Total-body PET/CT using half-dose FDG and compared with conventional PET/CT using full-dose FDG in lung cancer. Eur J Nucl Med Mol Imaging. 2020. 10.1007/s00259-020-05091-4.

10. Liu G, Hu P, Yu H, Tan H, Zhang Y, Yin H, et al. Ultra-low-activity total-body dynamic PET imaging allows equal performance to full-activity PET imaging for investigating kinetic metrics of 18F-FDG in healthy volunteers. Eur J Nucl Med Mol Imaging. 2021 Jan 22. doi: 10.1007/s00259-020-05173-3. Epub ahead of print. PMID: 33479842.

11. Boellaard R, Delgado-Bolton R, Oyen WJ, Giammarile F, Tatsch K, Eschner W, et al. FDG PET/CT: EANM procedure guidelines for tumour imaging: version 2.0. Eur J Nucl Med Mol Imaging. 2015;42(2):328-54. 10.1007/s00259-014-2961-x.

12. Tahari AK, Chien D, Azadi JR, Wahl RL. Optimum lean body formulation for correction of standardized uptake value in PET imaging. J Nucl Med. 2014;55(9):1481-4. doi: 10.2967/jnumed.113.136986.

13. Obesity: preventing and managing the global epidemic. Report of a WHO consultation. World Health Organ Tech Rep Ser. 
2000;894:i-xii, 1-253. PMID: 11234459.

14. Janmahasatian S, Duffull SB, Ash S, Ward LC, Byrne NM, Green B. Quantification of lean bodyweight. Clin Pharmacokinet. 2005;44(10):1051-65. 10.2165/00003088-200544100-00004.

15. de Groot EH, Post N, Boellaard R, Wagenaar NR, Willemsen AT, van Dalen JA. Optimized dose regimen for whole-body FDG-PET imaging. EJNMMI Res. 2013;12;3(1):63. doi: 10.1186/2191-219X-3-63.

16. Cox CPW, Segbers M, Graven LH, Brabander T, van Assema DME. Standardized image quality for 68Ga-DOTA-TATE PET/CT. EJNMMI Res. 2020;23;10(1):27. doi: 10.1186/s13550-020-0601-y.

17. Delbeke D, Coleman RE, Guiberteau MJ, Brown ML, Royal HD, Siegel BA, et al. Procedure guideline for tumor imaging with 18F-FDG PET/CT 1.0. J Nucl Med. 2006;47(5):885-95.

18. Mizuta T, Senda M, Okamura T, Kitamura K, Inaoka Y, Takahashi M, et al. NEC density and liver ROI S/N ratio for image quality control of whole-body FDG-PET scans: comparison with visual assessment. Mol Imaging Biol. 2009 ;11(6):480-6. doi: $10.1007 / \mathrm{s} 11307-009-0214-3$.

19. Büsing KA, Schönberg SO, Brade J, Wasser K. Impact of blood glucose, diabetes, insulin, and obesity on standardized uptake values in tumors and healthy organs on 18F-FDG PET/CT. Nucl Med Biol. 2013;40(2):206-13. doi: 10.1016/j.nucmedbio.2012.10.014.

20. Laffon E, Adhoute X, de Clermont H, Marthan R. Is liver SUV stable over time in ${ }^{18} \mathrm{~F}-\mathrm{FDG}$ PET imaging? J Nucl Med Technol. 2011;39(4):258-63. doi: 10.2967/jnmt.111.090027. 
Figures

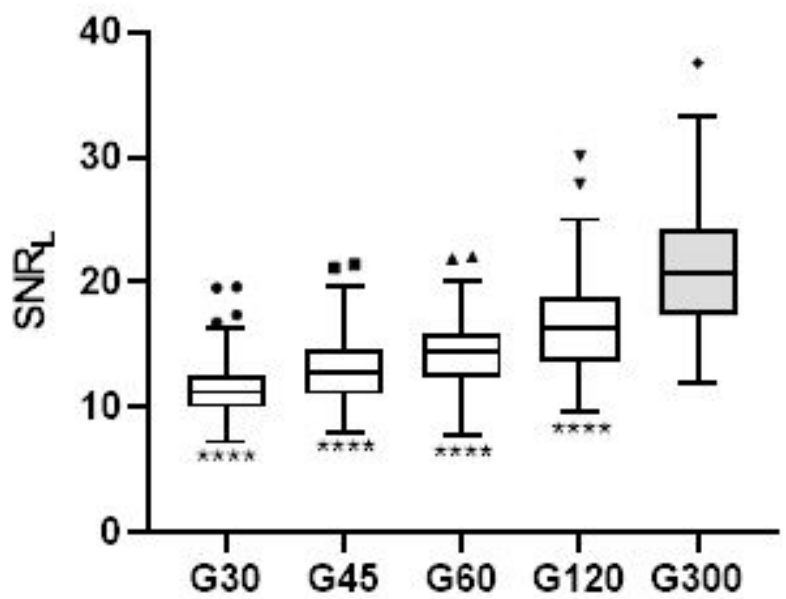

Figure 1

Boxplots of the SNRL (a) against acquisition durations. According to Whiskers specify Tukey test, a significant difference compared with G300 (Grey bar) is indicated with an asterisk $\left({ }^{*}, p<0.05\right.$; $* \star \star \star, p<$ 0.0001).
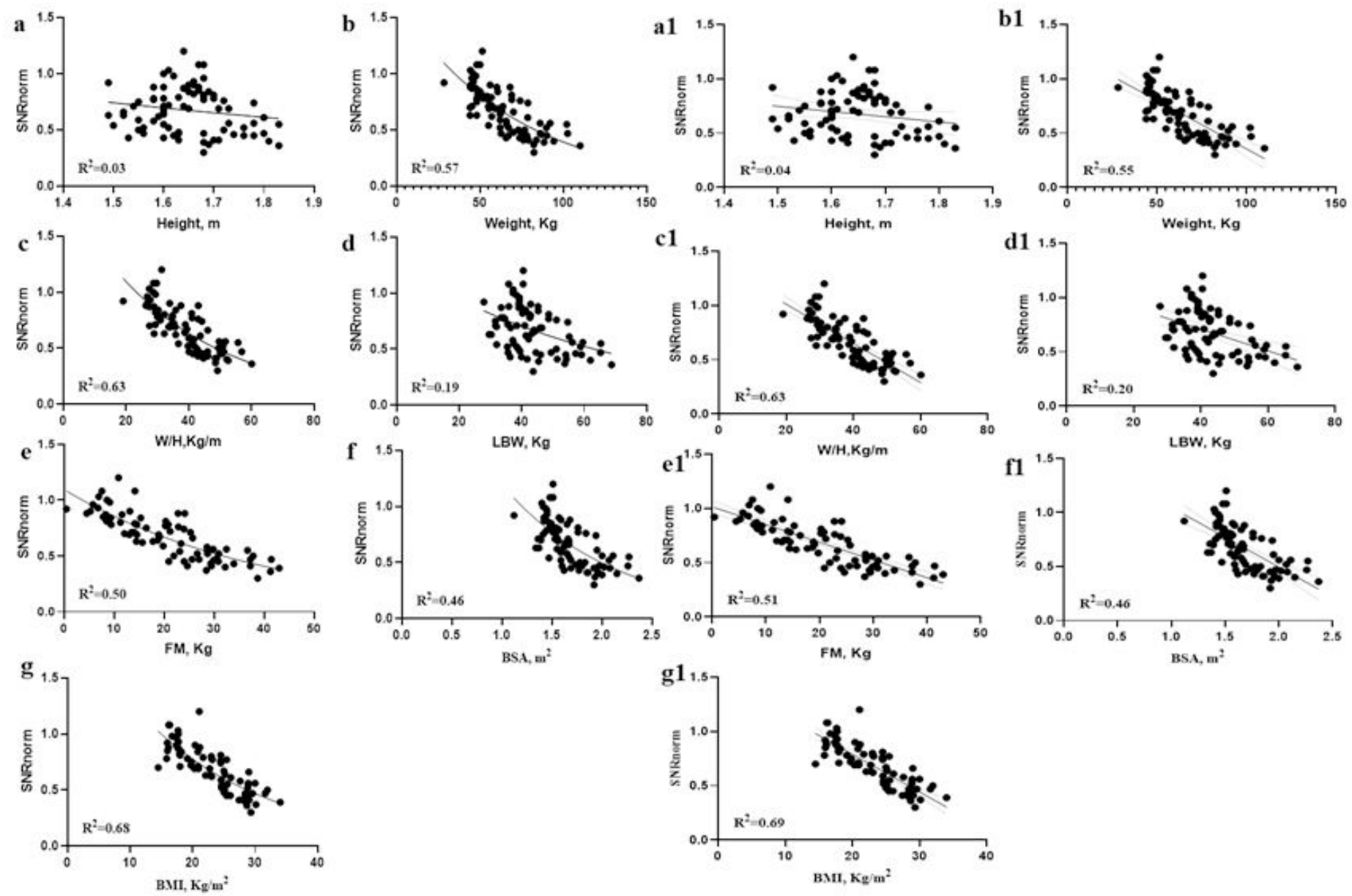


\section{Figure 2}

The linear fits ( $a-h)$ and non-linear fits (a1-h1) of SNRnorm against patient-dependent parameters. The parameter included height $(a, a 1)$, weight $(b, b 1)$, weigh per height $(W / H, c, c 1)$, lean body weight $(L B M, d$, d1), fat mass (FM, e, e1), body surface area (BSA, f, f1) and body mass index (BMI, g, g1).
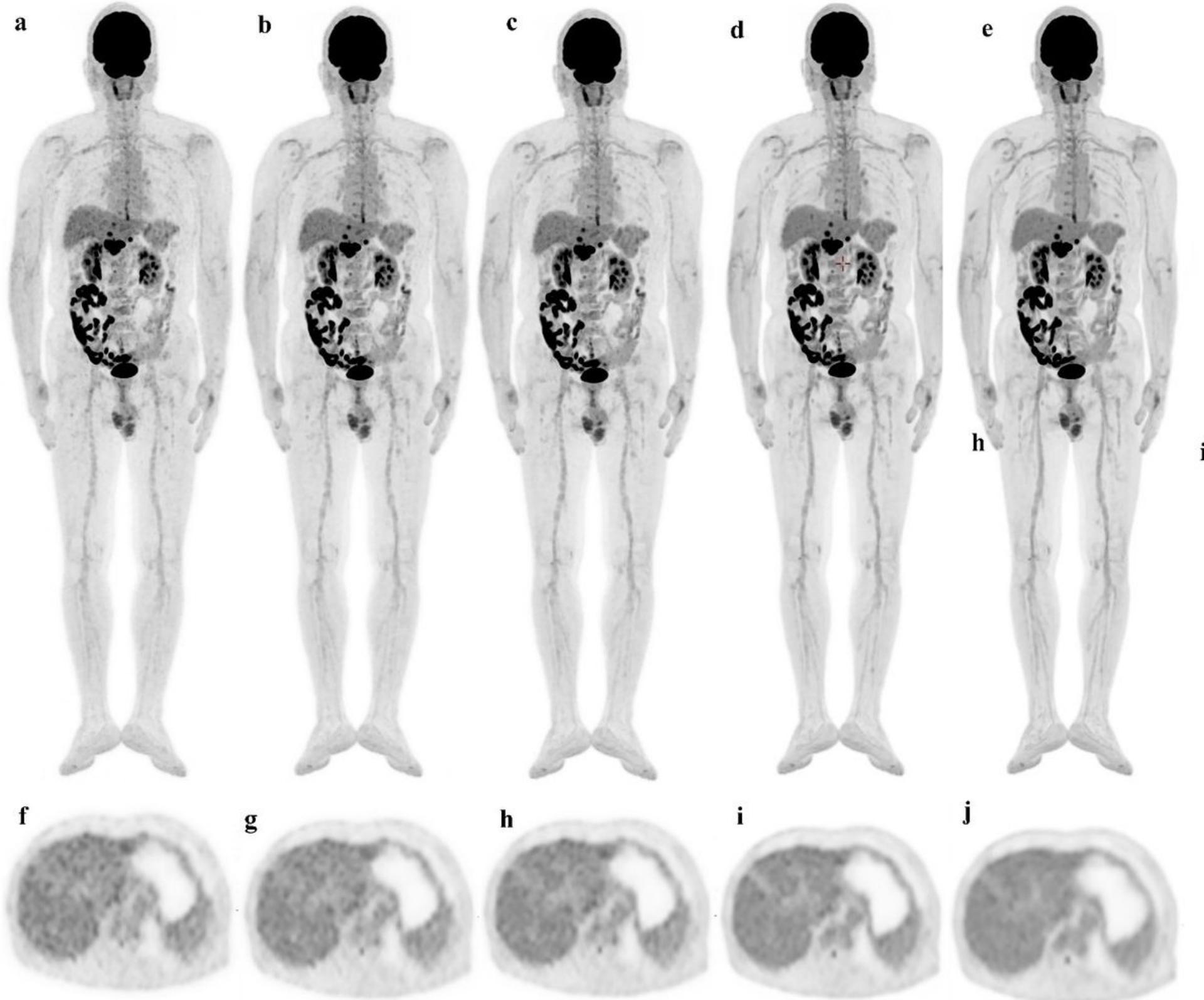

G30

G45

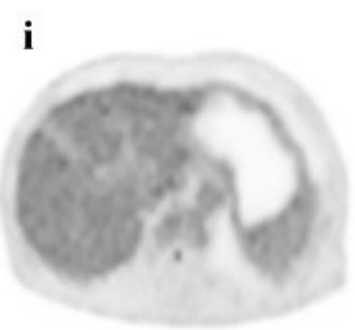

G120 j

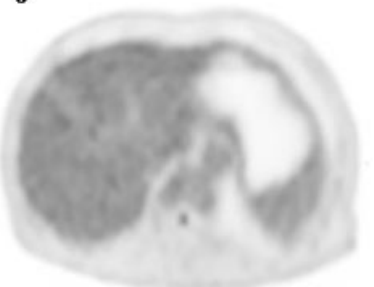

G300

Figure 3

An example of subjectively qualitative scores. The image quality score 2, 2, 3, 4 and 5 were given to the G30, G45, G60, G120 and G300, according to axial view images (a-e). 


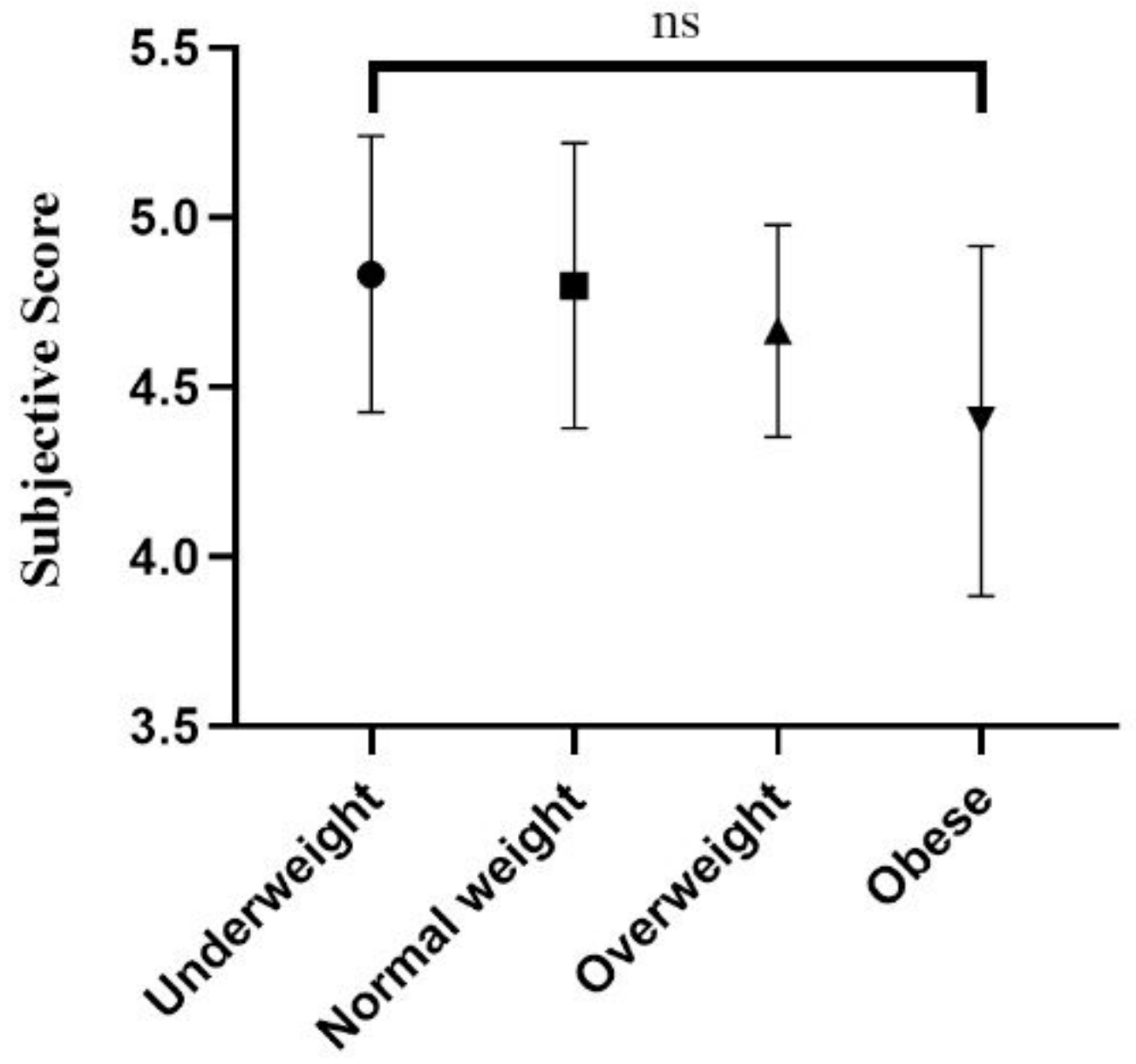

Figure 4

Subjective scores of different BMI groups in the validation cohort. There was no significant difference between the BMI groups. Note: ns=no significant difference. 
a
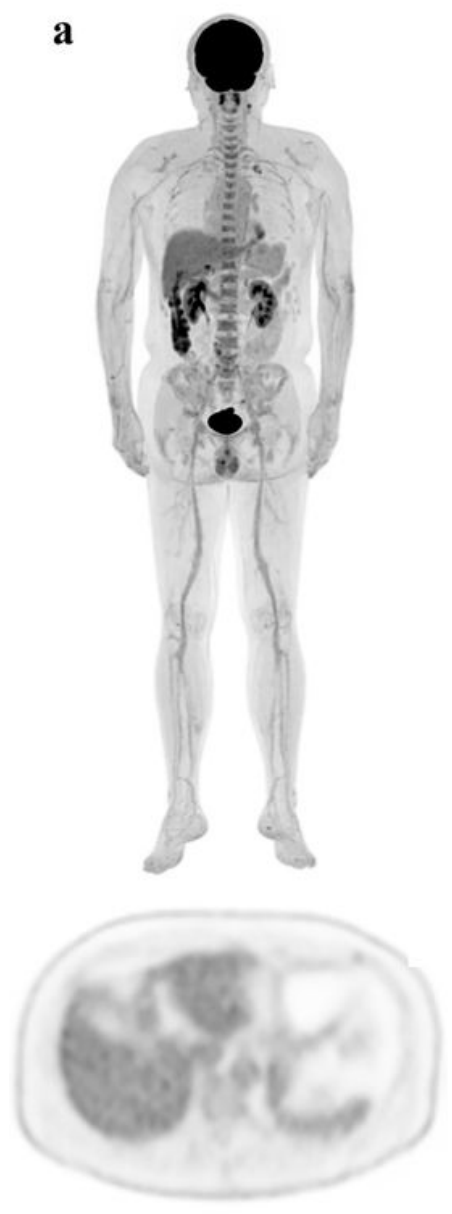

BMI $=25 \mathrm{~kg} / \mathrm{m}^{2}$

Dose $=278 \mathrm{MBq}$ b
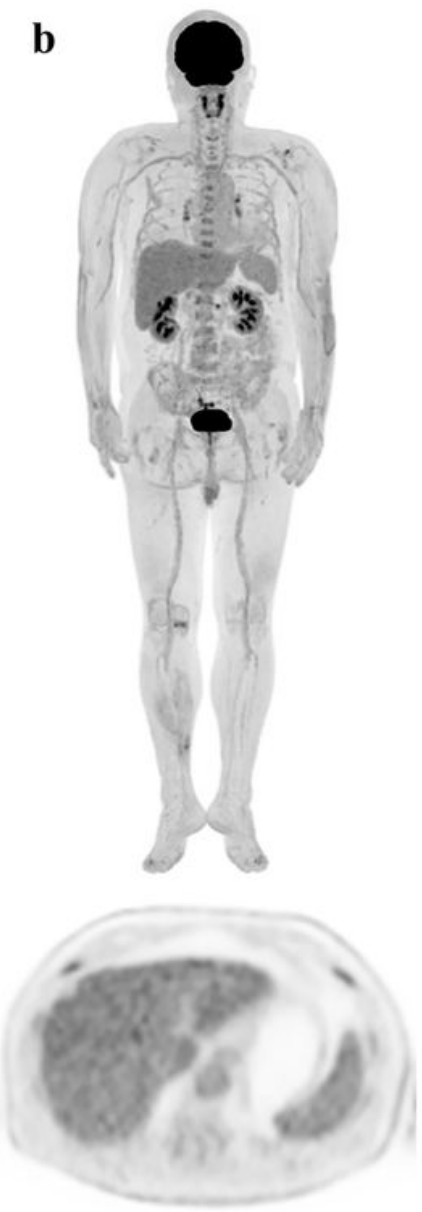

BMI $=30 \mathrm{~kg} / \mathrm{m}^{2}$

Dose $=370 \mathrm{MBq}$ c
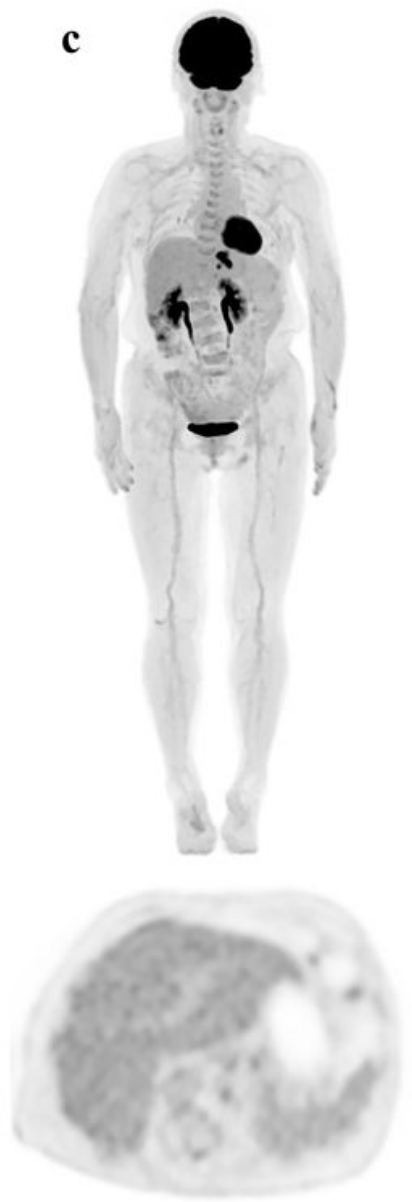

$\mathrm{BMI}=25 \mathrm{~kg} / \mathrm{m}^{2}$

Dose $=81 \mathrm{MBq}$ d
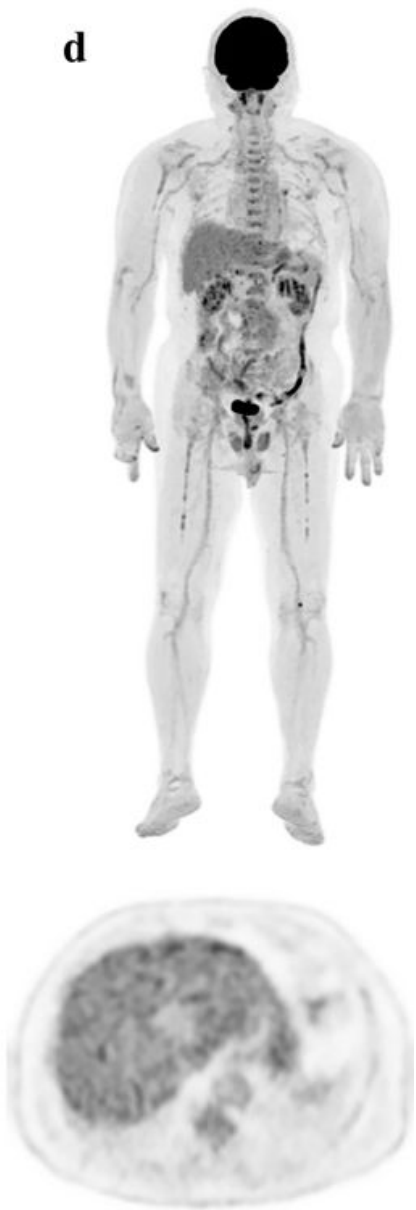

BMI $=30 \mathrm{~kg} / \mathrm{m}^{2}$

Dose $=108 \mathrm{MBq}$

Figure 5

Patient image in developing dataset and validation dataset. MIP and transverse images of total-body $18 F-F D G$ PET for Patient with BMI of $25 \mathrm{~kg} / \mathrm{m} 2$ and $30 \mathrm{~kg} / \mathrm{m} 2$ in full-dose regimen $(\mathrm{a}, \mathrm{b})$ and BMI-related dose regimen $(c, d)$. 
a

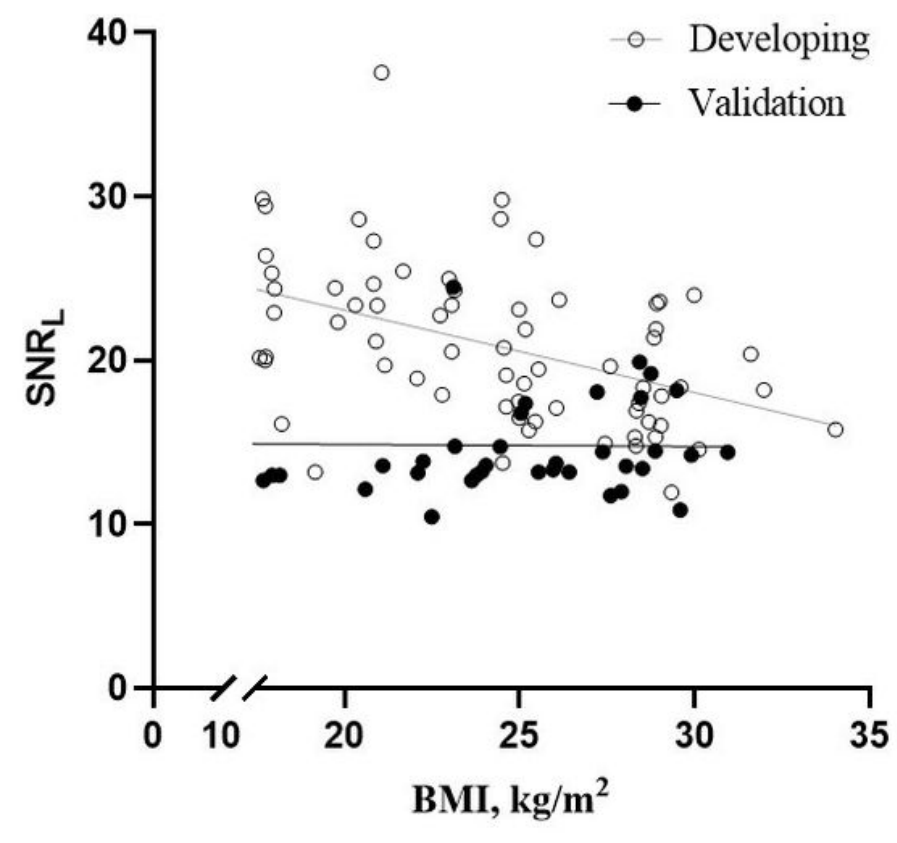

b

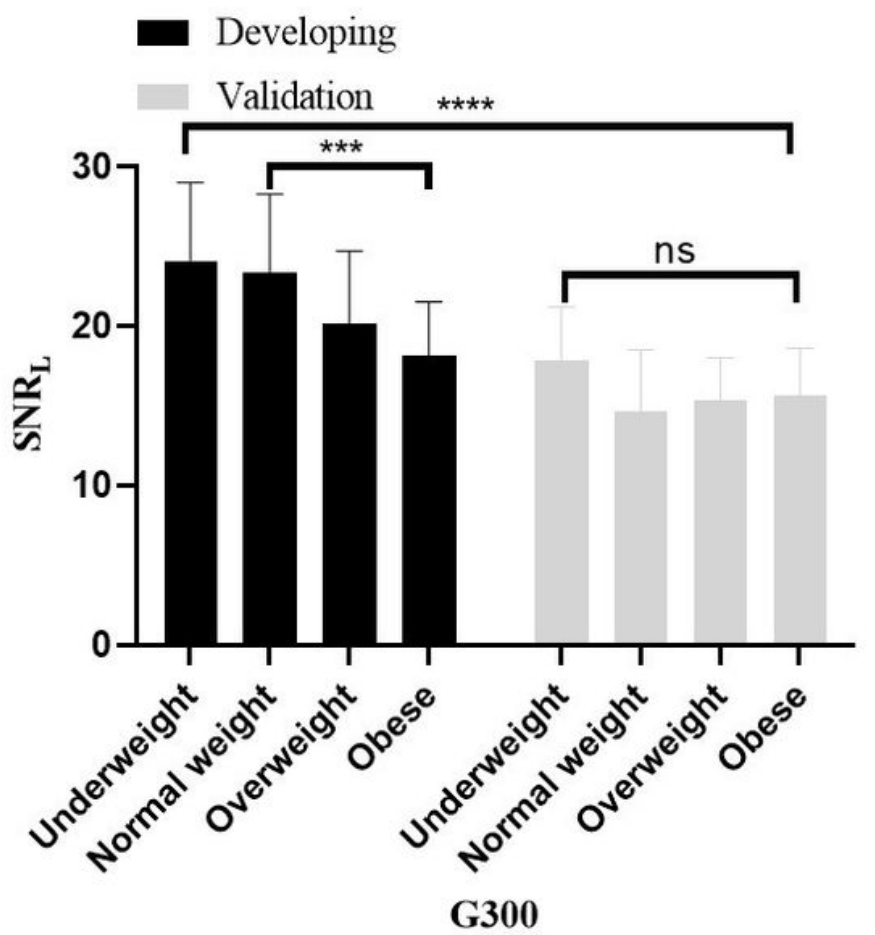

Figure 6

Comparison the differences of SNRL between BMI groups in developing dataset and validation dataset. The SNRL was negative correlation with BMI in developing dataset but not changed with BMI in validation dataset.

\section{Supplementary Files}

This is a list of supplementary files associated with this preprint. Click to download.

- SupplementaryInformationSI.docx 